\title{
Effects of Nd on Extrusion Microstructure and Mechanical Properties of ZK20 Magnesium Alloy
}

\author{
Quan $\mathrm{L}^{1} \mathrm{i}$, Weibo $\mathrm{Zhu}^{1}$, Xianquan Jiang ${ }^{1}$, Bin Zeng ${ }^{2}$, Fusheng Pan $^{2}$ \\ ${ }^{1}$ Chongqing Academy of Science and Technology \\ ${ }^{2}$ Chongqing Academy of Science and Technology and College of Materials Science \& Engineering, \\ Chongqing University
}

Keywords: Magnesium alloy; Homogenization; Microstructure; Extrusion.

\begin{abstract}
In this paper, adding different Nd content of ZK20 magnesium alloy extrusion were studied Nd on ZK20 magnesium alloy extrusion and mechanism of influence of microstructure and mechanical properties, and optimization of Nd in ZK20 magnesium alloy added amount, in order to obtain comprehensive mechanical performance optimization of alloys. The results showed that the rare earth neodymium organization can effectively refine ZK20 magnesium alloy extrusion, and can greatly improve the ductility properties of the alloy, which, when added in an amount of $0.5 \%$ neodymium, the grain most small and optimal mechanical proper-ties, but also by fracture brittle fracture into ductile fracture.
\end{abstract}

\section{Introduction}

Magnesium alloy due to low density, high strength, as the lightweight construction materials has broad application prospects. Wrought magnesium alloy cast product is relatively diverse and mechanical properties superior product shape and other characteristics, and gradually attracted much attention [7,8]. Magnesium belongs hcp lattice and plastic deformation capacity is less than aluminum and steel, increasing the difficulty and cost of preparation of the production of magnesium alloy plastic processing. Research and development of new magnesium alloys in recent years, it has been paying attention through the design and introduction of new alloy phase deformation process, etc., at the same time to ensure its strength to improve the processing of plastic deformation of magnesium alloys $[3,4,7]$. RE has a very beneficial effect for magnesium alloy, not only constitute the Mg-Re alloy system, but also Mg-Al, Mg-Zn alloy, both have a very significant impact, its main role: grain refinement, purification melting body, improve the alloy at room temperature strength, thermal stability, improve the mechanical properties of the alloy to improve the corrosion resistance of the alloy, etc $[1,2,5]$. In this paper, adding different Nd content of ZK20 magnesium alloy extrusion were studied Nd on ZK20 magnesium alloy extrusion and mechanism of influence of microstructure and mechanical properties, and optimization of Nd in ZK20 magnesium alloy added amount, in order to obtain comprehensive mechanical Performance optimization of alloys, and provide experimental evidence.

\section{Experiments}

Semi-continuous casting systems using water-cooled casting containing different concentrations of Nd of ZK20 magnesium alloy semi-continuous slab, the chemical composition of each alloy in Table 1 below. The resulting ingot casting in the wind circulation 12KW box-type resistance furnace homogenization treatment, air-cooled ingot after peeling, and extrusion tests on 500 tons of horizontal extruder, extrusion at $420^{\circ} \mathrm{C} \times 10 \mathrm{~h}$ blank in a mold heating furnace $2 \sim 3 \mathrm{~h}$ incubation in $400{ }^{\circ} \mathrm{C}$, extrusion cylinder setting temperature of $390{ }^{\circ} \mathrm{C}$, extrusion speed of $1 \sim 3 \mathrm{~m} / \mathrm{min}$. In the semi-continuous ingot samples $1 / 2$ radius cut by $5 \%$ ethanol solution of nitric acid corrosion, with OPTEC company MDS Watch microscope. The company uses TESCAN VEGA II LMU variable vacuum SEM and EDS were scanned and microstructure analysis of compound ingredients. Think of 
the new CMT-5105 electronic universal testing machine were room temperature tensile testing, tensile speed of $4 \mathrm{~mm} / \mathrm{min}$.

Table 1 Chemical compositions of experimental alloys (mass fraction, \%)

\begin{tabular}{|c|c|c|c|c|c|c|}
\hline Alloy code & $\mathrm{Si}$ & $\mathrm{Fe}$ & $\mathrm{Zn}$ & $\mathrm{Zr}$ & $\mathrm{Nd}$ & $\mathrm{Mg}$ \\
\hline ZK20 & 0.0063 & 0.0046 & 2.11 & 0.021 & 0.00 & Bal \\
\hline ZK20+0.1Nd & 0.0062 & 0.0038 & 2.05 & 0.023 & 0.09 & Bal \\
\hline ZK20+0.3Nd & 0.0061 & 0.0020 & 2.07 & 0.021 & 0.28 & Bal \\
\hline ZK20+0.5Nd & 0.0065 & 0.0018 & 2.06 & 0.062 & 0.47 & Bal \\
\hline ZK20+0.7Nd & 0.0058 & 0.0016 & 1.98 & 0.045 & 0.68 & Bal \\
\hline
\end{tabular}

\section{Test results and analysis}

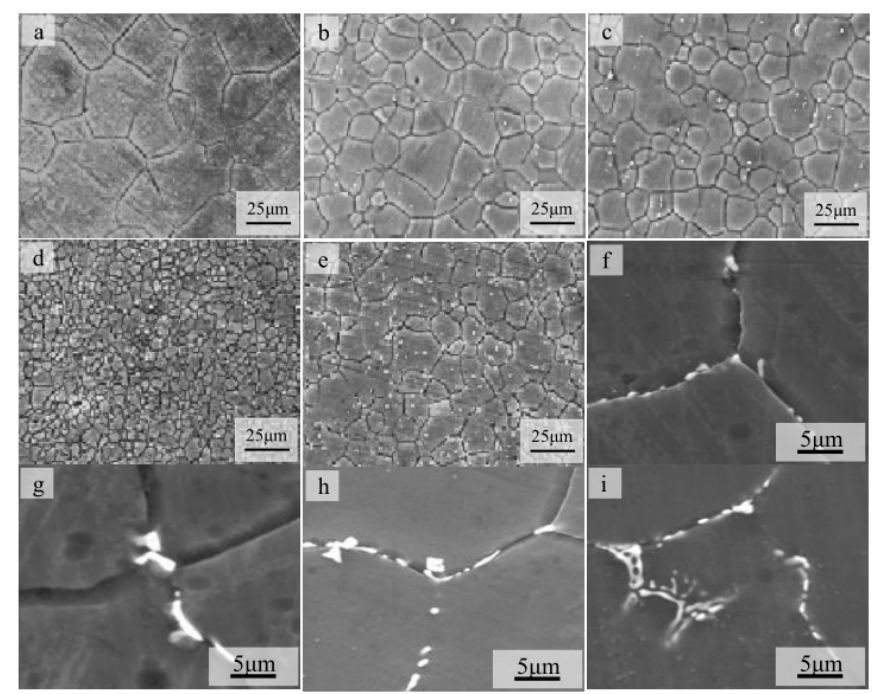

Fig1 Microstructure and the morphology of phases of the as-extruded alloy;

(a): Optical microstructure,ZK20 alloy, as-extruded;(b):Optical microstructure, ZK20+0.1Nd alloy, as-extruded;(c): Optical microstructure, ZK20+0.3Nd alloy ,as-extruded; (d): Optical microstructure, ZK20+0.5Nd alloy ,as-extruded;(e): Optical microstructure, ZK20+0.7Nd alloy ,as-extruded; (f): The phases, ZK20+0.1Nd alloy;(g): The phases, ZK20+0.3Nd alloy; (h): The phases, ZK20+0.5Nd alloy; (i): The phases, ZK20+0.7Nd alloy

Figure1 is extruded alloy microstructure and alloy phase morphology, the figure shows, the alloy dynamic recrystallization during extrusion, organizations are recrystallized fine equated, where the average state ZK20 alloy extrusion grain size is about $21.7 \mu \mathrm{m}$, neodymium add the extruded alloy microstructure is refined, to reduce the average grain size below $12 \mu \mathrm{m}$, refining effect is obvious. With the increase of the addition amount of neodymium, more and more fine crystal grains, and to achieve the smallest at $0.5 \% \mathrm{Nd}$, the grain size below $5 \mu \mathrm{m}$, neodymium element in the dynamic recrystallization from a good role, effectively refine grain, of which $0.5 \% \mathrm{Nd}$ alloy grain of most small and uniform. Alloy phase morphology can be seen from Fig1.After the addition of the alloy of $\mathrm{Nd}$ are present at grain boundaries are more granular fine alloy phase containing $\mathrm{Nd}$, they are uniformly distributed in the grain boundaries, most of the particle size of less than $2 \mu \mathrm{m}$, the small number of is the size of the larger particles and elongated, presumably these contain Nd alloy phase is an important reason alloy extrusion recrystallization refinement.

Extruded alloy mechanical properties shown in Figure 2, the figure shows, squeezing state ZK20 alloy tensile strength of 227MPa, yield strength of $139 \mathrm{MPa}$, elongation of $21.8 \%$. With the increase of the addition amount of neodymium, strength extruded alloys increased slightly, so that addition of $0.1 \%$ of neodymium is less than the tensile strength of the alloy to improve $4 \mathrm{MPa}$, adding $0.5 \%$ of neodymium alloy reaches the maximum tensile strength of 237 MPa, ZK20 alloy ratio increased about 5\%. Neodymium add more apparent yield strength increases, $0.1 \%-0.7 \%$ of the added amount 
of the alloy of neodymium yield strength increased to $150 \mathrm{MPa}$ above, increase the maximum rate of $10 \%$. Neodymium add the extruded plastic alloys have a more substantial increase, adding $0.1 \%$ neodymium alloy make elongation increased from 21 percent to about 30 percent, followed with increasing the added amount of neodymium, a slight increase in elongation When adding $0.5 \% \mathrm{Nd}$ alloy reaches the maximum elongation of 32.8\%, compared ZK20 alloy increased by more than 50\%. ZK20 and ZK20+0.5Nd cast alloy extrusion state performance and the performance of the control as shown in Figure 3, ZK20 alloy extruded after grain $238.8 \mu \mathrm{m}$ organized by the cast down to squeeze after $23.9 \mu \mathrm{m}$, squeeze performance than cast performance greatly improved, the tensile strength of the cast $187.5 \mathrm{MPa}$ grow to $227.4 \mathrm{MPa}$, extension rate cast $18 \%$ increase to $21.8 \%$. ZK20+0.5Nd cast alloy is $78.2 \mu \mathrm{m}$, after extrusion grain size $4.9 \mu \mathrm{m}$, cast extrusion performance ratio performance is also greatly improved, the tensile strength of the cast increased to $237.4 \mathrm{MPa}$, elongation rate cast $22.2 \%$ increase to $32.8 \%$, after ZK20 and ZK20+ $0.5 \mathrm{Nd}$ alloy extrusion yield strength has been greatly improved, the yield ratio alloys increase from about 0.3 to 0.6 above.

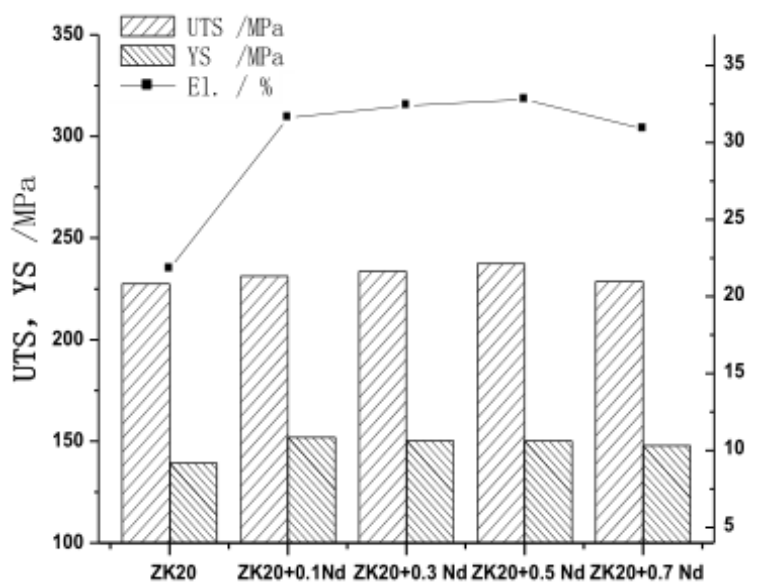

Fig.2 The tensile properties of as-extruded alloys

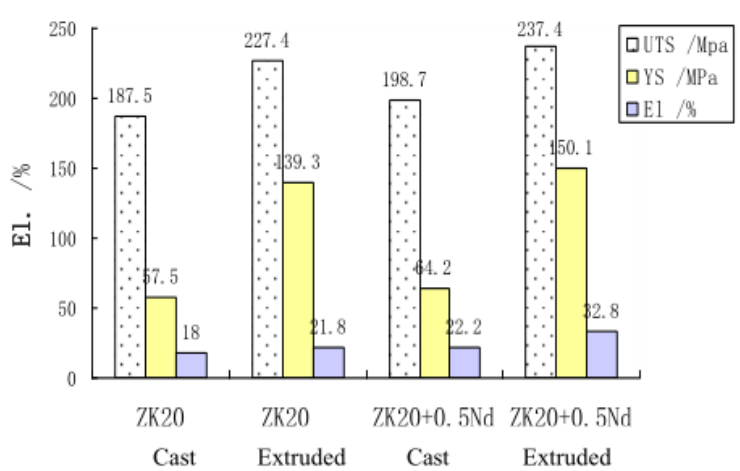

Fig.3 Fracture surface of tensile specimens

\section{Conclusions}

Adding rare earth neodymium make ZK20 alloy extrusion alloys organization effectively refine the average grain size decreased from $21.7 \mu \mathrm{m}$ to $12 \mu \mathrm{m}$ or less, with the increase of the added amount of neodymium, the grain is getting smaller, when the added amount of neodymium was $0.5 \%$, the most fine grain material.

Neodymium add strength on ZK20 magnesium alloy extruded product is not obvious, but it can improve the ductility of the alloy, and adding the alloy reaches the maximum elongation of $0.5 \%$ of Nd, than ZK20 alloy improved over 50\%.

Extruded ZK20+xNd tensile strength than cast alloy has a slight increase, leaving the elongation has increased greatly. Grain refinement makes alloy strength increases, but increased with the addition amount of neodymium, the matrix form a ternary alloy with zinc, zinc solid solution 
strengthening effect weakened, so that added to improve the strength of the alloy of neodymium role than enhance the role of plasticity outstanding.

Extruded ZK20 alloy fracture surface is rough, mainly by larger fracture cleavage fracture surface, the obvious parallel edges and very small tear-shaped dimples, belonging to brittle fracture based mixed fracture morphology. The increase of the added amount of neodymium, parallel tear-shaped ridge on the alloy fracture reduction blunt cleavage plane area is reduced, increasing the appearance of dimples, with mixed fracture into brittle fracture and fracture toughness; when neodymium added greater than $0.5 \%$, the fracture into ductile fracture based mixed fracture.

\section{Acknowledgements}

This work is supported by the International Science and Technology Cooperation Project (2013DFA71070, 2014DFG52810); Chongqing integrated demonstration projects (cstc2013jcsf 50003);

\section{References}

[1] Bussiba A, Ben Artzy A, Shtechman a et al, 2001. Grain refinement of AZ31 and ZK60 Mg alloys-towards super plasticity studies [J]. Materials Science and Engineering, A302:56\}62.

[2] Dale Latwell, Matthew R Barnett, 2007. Extrusion limits of magnesium alloys [J]. Metallurgical and Materials Transactions A, 38(12): 3032-3041.

[3] J.S. Zhang, J. Yan, W. Liang, C.X. Xu, C.L. Zhou 2008. Icosahedral quasicrystal phase in Mg-Zn-Nd ternary system [J]. Materials Letters, 62 (2008): 4489-4491.

[4] Li Wenxian, 2005. Magnesium and magnesium alloys [M]. Changsha: Central South University Press 6.

[5] Pan Fusheng, Han Enhou, 2007. High-Performance Wrought Magnesium Alloy and Their Processing Technigues [M]. Beijing: Science Press.18.

[6] Qiang Li, Qudong Wang, Haitao Zhou, Xiaoqing Zeng, Ya Zhang, Wenjiang Ding, 2005. High strength extruded $\mathrm{Mg}-5 \mathrm{Zn}-2 \mathrm{Nd}-1.5 \mathrm{Y}-0.6 \mathrm{Zr}-0.4 \mathrm{Ca}$ alloy produced by electromagnetic casting [J]. Materials Letters (59): 2549 - 2554.

[7] Qiang Li, Qudong Wang, Yingxin Wang, Xiaoqin Zeng, Wenjiang Ding, 2007. Effect of Nd and $\mathrm{Y}$ addition on microstructure and mechanical properties of as-cast Mg-Zn-Zr alloy. [J]. Journal of Alloys and Compounds (427): 115-123.

[8] Yu Kun, Li Wenxian, Zhang Shijun, 2005. Mechanism of Grain Refining by Adding Cerium in Mg and Mg Alloys [J]. Rare Metal Materials and Engineering, Vol. 34 (7):1013-1016. 